\title{
Avaliação dos efeitos da liberação miofascial instrumental sobre o músculo diafragma
}

\author{
Evaluation from the effects of the myofascial release over the diaphragm muscle \\ Evaluación de los efectos de la liberación miofascial instrumental sobre el músculo diafragma
}

Recebido: 04/10/2021 | Revisado: 10/10/2021 | Aceito: 14/10/2021 | Publicado: 17/10/2021

\author{
Caroline Gomes Nicorena \\ ORCID: https://orcid.org/0000-0003-4443-5631 \\ Universidade Federal do Pampa, Brasil \\ E -mail: carolinnegomesp@gmail.com \\ Leandro da Silva Peireira \\ ORCID: https://orcid.org/0000-0003-4839-1728 \\ Universidade Federal do Pampa, Brasil \\ E-mail: leandro25spereira@yahoo.com.br \\ Jéssica Delamuta Vitti \\ ORCID: https://orcid.org/0000-0003-1987-3891 \\ Hospital da Mulher Prof. Dr. José Aristodemo Pinotti, Brasil \\ E-mail: jehvitti@hotmail.com \\ Maurício Tatsch Ximenes Carvalho \\ ORCID: https://orcid.org/0000-0001-6679-2854 \\ Centro Universitário da Região da Campanha, Brasil \\ E-mail: mauximen96@hotmail.com \\ Antônio Adolfo Mattos de Castro \\ ORCID: https://orcid.org/0000-0002-7323-0937 \\ Universidade Federal do Pampa, Brasil \\ E-mail: antoniocastro@unipampa.edu.br \\ Gustavo Pilon \\ ORCID: https://orcid.org/0000-0003-4366-2330 \\ Clínica de quiropraxia \& eletro-acupuntura corporal, Brasil \\ E-mail: gustavopilon@gmail.com \\ Nelson Francisco Serrão Júnior \\ ORCID: https://orcid.org/0000-0002-0280-0752 \\ Universidade Federal do Pampa, Brasil \\ E-mail: nelsonserrao@unipampa.edu.br
}

\begin{abstract}
Resumo
Objetivo: Avaliar os efeitos da Instrument Assist Soft Tissue Mobilization (IASTM) Conceito Gustavo Pilon, o método instrumental de manipulação miofascial, sobre o músculo diafragma. Metodologia: o estudo foi realizado em uma voluntária, sexo feminino, idade de 21 anos, residente da cidade de Uruguaiana/RS. A mesma foi submetida a avaliação de força dos músculos respiratórios e avaliação ultrassonográfica do diafragma antes e após a intervenção, na qual foram aplicadas as técnicas do método de liberação miofascial instrumental, Conceito Gustavo Pilon. Resultados: Em relação à força muscular respiratória, traduzida pela Pressão Inspiratória Máxima (Pimáx) e Pressão Expiratória Máxima (Pemáx), verificou-se, respectivamente nos 3 momentos (M1, M2 e M3): Pimáx de 119cmH2O, $95 \mathrm{cmH} 2 \mathrm{O}$ e $95 \mathrm{cmH} 2 \mathrm{O}$; Pemáx de $112 \mathrm{cmH} 2 \mathrm{O}, 102 \mathrm{cmH} 2 \mathrm{O}$ e $109 \mathrm{cmH} 2 \mathrm{O}$. Já em relação à espessura muscular (EM), foram realizadas três medidas em cada momento, a saber: M1 com EM de 2,4cm, 2,4mm e 2,4cm; M2 com EM de 2,4cm, 2,4cm e 2,3cm; e M3 com EM de 2,8cm, 2,8cm e 2,8cm. Conclusão: No presente estudo não houve aumento da força muscular respiratória entre os momentos avaliados. Já em relação à espessura diafragmática, em apenas uma intervenção, verificou-se melhora do comprimento do músculo diafragma.
\end{abstract}

Palavras-chave: Fáscia; Manipulações musculoesqueléticas; Diafragma; Modalidades de fisioterapia.

\begin{abstract}
Objective: Evaluate the effects from the Instrument Assist Soft Tissue Mobilization (IASTM) Gustavo Pilon Concept, the instrumental method of myofascial manipulation, over the diaphragm muscle. Methodology: study accomplished with a volunteer from the feminine sex, with 21 years old, resident in the Uruguaiana/RS city. She underwent respiratory muscle strength assessment and a diaphragm ultrasound evaluation, before and after the intervention, in which were applied the myofascial instrumental release method technique, Gustavo Pilon Concept. Results: Regarding the respiratory muscle strength, translated by the Maximum Inspiratory Pressure (MIP) and the maximal expiratory pressure (MEP), it was verified, respectively at 3 momentums (M1, M2, M3): MIP of $119 \mathrm{cmH} 2 \mathrm{O}, 95 \mathrm{cmH}$ $2 \mathrm{O}$ and $95 \mathrm{cmH} 2 \mathrm{O}$; MEP of $112 \mathrm{cmH} 2 \mathrm{O}, 102 \mathrm{cmH} 2 \mathrm{O}$ and $109 \mathrm{cmH} 2 \mathrm{O}$. Furthermore, in relation to the Muscle
\end{abstract}


Thickness (MT), where formed 3 measures in each momentum, to know: M1 as MT of 2,4cm, 2,4mm and 2,4cm; M2 as MT of 2,4cm, 2,4cm and 2,3cm; also M3 as MT of 2,8cm, 2,8cm and 2,8cm. Conclusion: In the present study there was no increase in the respiratory muscle strength between the evaluated momentums. Regarding the diaphragm thickness, in only one intervention, was verified an improvement in the diaphragm muscle length.

Keywords: Fascia; Musculoskeletal manipulations; Diaphragm; Physical therapy modalities.

\section{Resumen}

Objetivo: Evaluar los efectos del concepto Gustavo Pilon de la Movilización de Tejidos Blandos con Instrumentos Asistidos (IASTM), el método instrumental de manipulación miofascial, en el músculo diafragma. Metodología: el estudio se realizó en una voluntaria, mujer, de 21 años, residente de la ciudad de Uruguaiana / RS. Fue sometida a valoración de la fuerza muscular respiratoria y valoración ecográfica del diafragma antes y después de la intervención, en la que se aplicaron las técnicas del método instrumental de liberación miofascial, Concepto Gustavo Pilón. Resultados: En cuanto a la fuerza de los músculos respiratorios, traducida por Presión máxima inspiratoria (Pimax) y Presión máxima espiratoria (Pemax), se verificó, respectivamente, en los 3 momentos (M1, M2 y M3): Pimax de $119 \mathrm{cmH} 2 \mathrm{O}, 95 \mathrm{cmH} 2 \mathrm{O}$ y 95cmH2O; Pmax de $112 \mathrm{cmH} 2 \mathrm{O}, 102 \mathrm{cmH} 2 \mathrm{O}$ y $109 \mathrm{cmH} 2 \mathrm{O}$. En relación al grosor muscular (EM), se tomaron tres medidas en cada momento, a saber: M1 con EM de 2,4cm, 2,4mm y 2,4cm; M2 con MS de 2,4 $\mathrm{cm}, 2,4 \mathrm{~cm}$ y 2,3 cm; y M3 con MS de 2,8 cm, 2,8 cm y 2,8 cm. Conclusión: En el presente estudio, no hubo aumento de la fuerza de los músculos respiratorios entre los momentos evaluados. En relación al grosor del diafragma, en una sola intervención, hubo una mejora en la longitud del músculo diafragma.

Palabras clave: Fascia; Manipulaciones musculoesqueléticas; Diafragma; Modalidades de fisioterapia.

\section{Introdução}

A fáscia é um tecido conjuntivo fibroso que envolve os músculos (Alves, Lins, Barros, \& Farah, 2008), o qual interliga todas as partes do corpo entre si como uma rede contínua de tecido, com propriedades biomecânicas significativas, auxiliando na manutenção da força muscular. O enfraquecimento do tecido fascial está associado a esforços excessivos, péssimos ambientes ergonômicos, traumas e alinhamento postural anormal, o que pode levar a alteração dos músculos ventilatórios, em especial, o diafragma. Esta atenuação do tecido acarreta uma rigidez, que causa dor e restringe os movimentos fasciais (De Domenico, 2015).

A liberação miofascial é uma técnica de terapia manual que atua mobilizando a fáscia manualmente ou com instrumentos, aplicando um alongamento de longa duração e baixa carga ao complexo miofascial (Ajimsha, AL-Mudahka, \& Al-Madzhar, 2015). A liberação miofascial tem como princípio proporcionar uma melhor interação entre fáscia e músculos estimulando a mobilidade do músculo tratado, e até mesmo de prevenir lesões musculares, sendo utilizada para quebrar aderências da fáscia e do tecido muscular que a envolve, principalmente do músculo diafragma (Sutton, B., 2016).

O diafragma é o principal músculo da respiração, sua forma curvilínea lhe confere grande força de contração, além de ser responsável por cerca de 60 a $70 \%$ do volume corrente durante uma respiração em repouso. Acredita-se que alterações na fáscia do músculo diafragma interferem diretamente na função respiratória, alterando a mobilidade diafragmática, força de contração, volumes e capacidades pulmonares (Kendall, Mccreary, \& Provance, 1995).

A realização da técnica de liberação miofascial utilizando instrumentos possui a mesma finalidade da técnica realizada de forma manual, porém oferece vantagem mecânica ao profissional. O uso dos raspadores na técnica liberação miofascial tem mostrado grandes benefícios na prática clínica, porém ainda são poucos os estudos na literatura que abordam os efeitos da liberação miofascial instrumental no músculo diafragma em indivíduos saudáveis, levando em consideração as medidas de força muscular respiratória e mobilidade dia (Henrique, Pfister, \& Ferreira, 2019).

A pressão inspiratória máxima (Pimáx) e a pressão expiratória máxima (Pemáx) têm sido consideradas, desde as décadas de 60 e 70, como um método simples, prático e preciso na avaliação da força dos músculos respiratórios, tanto em indivíduos sadios como em pacientes com disfunção respiratória ou neurológicas (Costa, Sampaio, Lorenzzo, Jamami, \& Damaso, 2003). Podem ser mensuradas através do manovacuômetro, um aparelho que tem por finalidade quantificar as pressões respiratórias positivas (manômetro) e pressões negativas (vacuômetro) Novaes, Sanchez \& Sanchez, 2013). 
A ultrassonografia permite medir a mobilidade da cúpula diafragmática e a espessura diafragmática na sua região de aposição ao gradil costal. A literatura demonstrou que a ultrassonografia diafragmática é uma ferramenta útil para avaliação da mobilidade do músculo, de forma não invasiva, livre de radiação e disponível nos hospitais, permitindo avaliações repetidas (Caruso, Albuquerque, Santana, Cardenas, Ferreira Prina et al 2015). A avaliação ultra-sônica do espessamento do diafragma tem sido descrita como um método não invasivo e reprodutível à beira do leito. A avaliação da espessura do diafragma na zona de aposição durante o ciclo respiratório fornece informações específicas sobre a força muscular. A fração de espessamento (FE) do diafragma foi proposta como um critério substituto para estimar a carga de trabalho respiratório (Moury, Cuisinier, Durand, Bosson, Chavanon, Payen, Jaber, \& Albaladejo, 2019).

A técnica de liberação miofascial visa liberar qualquer aderência da fáscia na musculatura, permitindo assim, que o movimento ocorra de forma harmônica em sua amplitude ideal e com a distribuição de forças de maneira correta (Pavan, Pachera, Forestiero, \& Natali, 2017). Portanto, o objetivo deste estudo foi demonstrar os efeitos da liberação miofascial instrumental sobre o músculo diafragma, avaliando a força muscular respiratória e avaliação ultrassonográfica do diafragma.

\section{Metodologia}

A presente pesquisa trata-se de um estudo de caso, com análise quantitativa (Pereira et al 2018), realizado em uma voluntária, sexo feminino, idade de 21 anos, residentes da cidade de Uruguaiana/RS. A mesma foi submetida a avaliação de força dos músculos respiratórios, realizada através do Manovacuômetro da marca Globalmed, modelo MVD 300 e avaliação ultrassonográfica (marca Chison, modelo EBIT30) do diafragma antes e após a intervenção.

As avaliações foram realizadas em 3 momentos(M): Momento 01 (M1): avaliação da força muscular respiratória e da espessura muscular do diafragma, após 2 minutos de repouso, foram aplicadas as técnicas do método de liberação miofascial instrumental (IASTM), Conceito Gustavo Pilon. Momento 2 (M2): avaliação de força dos músculos respiratórios e avaliação ultrassonográfica do diafragma 1 minuto após a intervenção. Momento 03 (M3), avaliação de força dos músculos respiratórios e avaliação ultrassonográfica do diafragma 5 minutos após a intervenção.

$\mathrm{Na}$ intervenção foram aplicadas as técnicas do conceito IASTM Gustavo Pilon, na qual foram utilizados os instrumentos do kit IASTM Gustavo Pilon ${ }^{\circledR}$. Os instrumentos são ergonomicamente projetados e constituídos de polietileno, resistentes a altas temperaturas, atóxicos, impermeáveis e apresentam alta resistência a tensão, compressão e tração.

A intervenção iniciou-se pela técnica de deslizamento que é aplicada no sentido longitudinal e transversal das fibras musculares, na qual, a pressão do raspador associada a baixa velocidade, promove redução da tensão das fibras e adicionado um estiramento o que proporciona um ganho na elasticidade do tecido. Logo depois foi utilizada a técnica oscilatória que segue o princípio da fáscia musculoesquelética, com o princípio de liberar aderências fasciais, otimizando o deslizamento do músculo diafragma na respiração. Portanto, todas as técnicas que foram aplicadas visavam, através da reprodução mecânica dos instrumentos, causar oscilação no tecido fascial e liberar sua movimentação natural.

A avaliação da espessura muscular diafragmática foi baseada em alguns estudos (Grosu, Lee, Lee, Eden, Eikermann \& Rose, 2012; Mccool, Benditt, Conomos, Anderson, Sherman \& Hoppin, 1997; Schepens, Verbrugghe, Dams, Corthouts, B., Parizel, \& Jorens, 2015). O sujeito foi mantido em decúbito dorsal, sequencialmente o transdutor do ultrassom, revestido com gel ultrassônico, foi posicionado no oitavo espaço intercostal no nível da linha axilar média (zona de aposição). O hemidiafragma direito foi visualizado, dinamicamente, no momento da inspiração como uma estrutura superficial obliterada pela borda pulmonar e no final da expiração como três camadas acima do fígado (uma estrutura muscular hipoecogênica, delimitada pela membrana peritoneal e a pleura diafragmática). A mensuração da espessura do diafragma foi feita no final da expiração. Os valores de espessura do diafragma foram gerados considerando a média de três medidas ultrassonográficas com uma diferença máxima entre elas de $0,1 \mathrm{~mm}$. 
O projeto foi aprovado pelo Comitê de Ética em Pesquisa da Fundação Universidade Federal do Pampa $\left(\mathrm{N}^{\circ}\right.$ parecer 3.704.014). As avaliações foram iniciadas após a assinatura do Termo de Consentimento Livre Esclarecido.

\section{Resultados}

Em relação à força muscular respiratória, traduzida pela Pressão Inspiratória Máxima (Pimáx) e Pressão Expiratória Máxima (Pemáx), ambas mensuradas em centímetros de água (cmH2O), verificou-se, respectivamente nos 3 momentos (M1, M2 e M3) os seguintes valores, conforme a Tabela 1:

Tabela 1 - Valores da Força Muscular Respiratória nos três momentos avaliados.

\begin{tabular}{cccccc}
\hline FMR & M1 & M2 & $\begin{array}{c}\text { Delta (M1-M2) } \\
\text { Delta (pós-pré)x 100(- } \\
\end{array}$ & \multicolumn{4}{c}{ M3 } & $\begin{array}{c}\text { Delta (M2-M3) } \\
\text { Delta (pós-pré)x 100(-1) }\end{array}$ \\
\hline \hline Pimáx $\left(\mathrm{cmH}_{2} \mathrm{O}\right)$ & $119 \mathrm{cmH}_{2} \mathrm{O}$ & $95 \mathrm{cmH}_{2} \mathrm{O}$ & $20 \%$ & $95 \mathrm{cmH}_{2} \mathrm{O}$ & $0 \%$ \\
Pemáx $\left(\mathrm{cmH}_{2} \mathrm{O}\right)$ & $112 \mathrm{cmH}_{2} \mathrm{O}$ & $102 \mathrm{cmH}_{2} \mathrm{O}$ & $9 \%$ & $109 \mathrm{cmH}_{2} \mathrm{O}$ & $6,4 \%$ \\
\hline
\end{tabular}

Legenda: FMR - Força Muscular Respiratória; $\mathrm{cmH}_{2} \mathrm{O}$ - centímetros de água; M1 - Momento 1; M2 - Momento 2; M3 - Momento 3. Fonte: Autores.

Já em relação à espessura muscular (EM), foram realizadas três medidas em cada momento, conforme a Tabela 2, a saber:

Tabela 2 - Avaliação da espessura muscular (EM) do músculo diafragma, nos três momentos avaliados.

\begin{tabular}{cccc}
\hline & $\begin{array}{c}\text { EM (cm) } \\
\text { M1 }\end{array}$ & $\begin{array}{c}\text { EM (cm) } \\
\text { M2 }\end{array}$ & $\begin{array}{c}\text { EM (cm) } \\
\text { M3 }\end{array}$ \\
\hline \hline M1 & 2,4 & 2,4 & 2,4 \\
M2 & 2,4 & 2,4 & 2,3 \\
Delta (M1-M2) & $0 \%$ & $0 \%$ & $4,2 \%$ \\
Delta (pós-pré) x100(-1) & 2,8 & 2,8 & 2,8 \\
M3 & $14,30 \%$ & $14,30 \%$ & $17,85 \%$ \\
Delta (M2-M3) & & & \\
Delta (pós-pré)x100(-1) & & & \\
\hline
\end{tabular}

Legenda: EM - espessura muscular; cm - centímetros; M1 - Momento 1; M2 - Momento 2; M3 - Momento 3.

Fonte: Autores.

De acordo com as variáveis não houve aumento da força muscular respiratória entre os momentos avaliados. Já em relação à espessura diafragmática, em apenas uma intervenção, verificou-se aumento na espessura muscular do diafragma.

\section{Discussão}

Salienta-se o ineditismo deste estudo, uma vez que é a primeira vez que um trabalho reflete e infere a melhora da mobilidade diafragmática pelo aumento da espessura muscular com a técnica de liberação miofascial instrumental (IASTM), Conceito Gustavo Pilon, em apena uma sessão. 
Segundo Hammer (2003), as fáscias podem apresentar tensões e começar a se endurecer, e aos poucos, essa rigidez se dissipa, fazendo com que a flexibilidade e a fluidez dos movimentos sejam perdidas, resultando em dores e limitação de movimento. Goetten (2018) em uma revisão da literatura sobre os efeitos da liberação miofascial corrobora afirmando que essas restrições miofasciais, acabam comprimindo os compartimentos fasciais e dificultam tanto na chegada de nutrientes, como na saída de metabólitos, ou seja, o músculo sujeito a restrições de movimento sofre de falta de sangue, além de acumular resíduos.

Foram encontrados na literatura estudos que utilizaram a liberação manual do diafragma, como no estudo de Rocha, Souza, Brandao, Rattes, Ribeiro, Campos e Andrade (2015) que avaliaram a força muscular respiratória após intervenção com liberação manual do diafragma, realizado em 20 pacientes portadores de Doença Pulmonar Obstrutiva Crônica (DPOC). Foram observadas melhorias significativas na mobilidade diafragmática, na capacidade de exercício, porém assim como o presente estudo não foram encontrados benefícios agudos em relação a força muscular respiratória. Porém, o estudo corrobora aos achados desta pesquisa em relação a mobilidade do diafragma, quando houve aumento da espessura do diafragma, principalmente entre M1 e M3.

A utilização da Instrument Assist Soft Tissue Mobilization (IASTM), o Conceito Gustavo Pilon, tem se mostrado eficaz em várias lesões e distúrbios na prática clínica, porém este é o primeiro estudo que abordou seus efeitos na força e na mobilidade do músculo diafragma. Esperava-se que através da aplicação do método IASTM o sujeito demonstrasse um aumento na força dos músculos respiratórios, entretanto, devido ao fato de ser apenas uma pessoa avaliada e somente após uma única sessão com intervenção e avaliação, nesse estudo não foi possível averiguar que a utilização do IASTM é eficaz na força muscular inspiratória.

Verificou-se no presente estudo aumento da força muscular expiratória, traduzida pelo aumento da Pemáx, evidenciando que conforme há alteração da espessura diafragmática (musculatura inspiratória), isso possa alterar a mecânica ventilatória e, consequentemente, o padrão expiratório. O mesmo foi evidenciado no estudo realizado por Novaes, Sanchez e Sanchez (2013) que compararam as pressões respiratórias em homens e mulheres sedentários antes e depois da técnica de liberação diafragmática, e demonstraram um aumento significativo da Pemáx depois da intervenção, representando um aumento da força dos músculos expiratórios, dados estes que corroboram ao resultado de nosso estudo piloto.

Braga, Marizeiro, Florêncio, Teles, Silva, Santos-Júnior e Campos (2020) utilizaram a terapia manual no diafragma com o objetivo de avaliar a força muscular respiratória e na mobilidade torácica em indivíduos jovens e saudáveis, mostrandose também consistente com os achados de nossa pesquisa referente ao aumento da Pemáx.

O estudo realizado por Moreno, Catai, Teodori, Borges, Cesar e Silva (2007) mostram que o diafragma é responsável por $60 \%$ a $70 \%$ do volume corrente nas respirações normais e as disfunções no tecido fascial nesse músculo, podem causar variações nas pressões respiratórias em indivíduos saudáveis. Novaes, Sanchez e Sanchez (2013) ressaltam que o diafragma desempenha um papel de essencial na respiração e a importância de uma intervenção fisioterapêutica quando o mesmo se mostra ineficaz ou fraco. Nair, Alaparthi, Krishnan, Rai, Anand, Acharya e Acharya (2019) utilizaram a técnica de liberação manual do diafragma em 20 pacientes portadores de DPOC e demonstraram que a técnica proporcionou uma melhora na flexibilidade dos músculos respiratórios, trazendo uma melhora na relação comprimento-tensão, melhorando assim o desempenho da mecânica respiratória.

Dado o efeito benéfico observado na espessura diafragmática, pode-se evidenciar que o presente estudo mostrou através de uma avaliação quantitativa da zona de aposição do diafragma, que as técnicas de liberação miofascial utilizando IASTM, Conceito Gustavo Pilon, promoveram a liberação eficaz da fáscia, alongando essa musculatura, aumentando a espessura do músculo diafragma, inferindo aumento na mobilidade do músculo, trazendo benefícios para na mecânica respiratória e ventilatória dessa participante. 
Como limitação do estudo, a presente pesquisa foi prejudicada pela pandemia do covid-19 e por este fato foi realizada com apenas um sujeito, mas destacamos a importância da realização de um estudo que possa contar com uma amostra fidedigna para demonstrar os efeitos da técnica de liberação miofascial instrumental (IASTM), Conceito Gustavo Pilon, quando aplicada no músculo diafragma.

\section{Conclusão}

Após explanação dos resultados, verificou-se que não houve melhora da força muscular respiratória entre os momentos avaliados. Já em relação à espessura diafragmática, em apenas uma intervenção, verificou-se melhora do comprimento do músculo diafragma.

Novos estudos devem ser realizados com temática da avaliação da força muscular respiratória, com maior número de participantes, a fim de aprimorar as condutas e atendimentos aos pacientes, minimizando os riscos e permitindo uma melhor reabilitação. A partir dos dados coletados nessa pesquisa, almeja-se colaborar com futuros estudos sobre o tema, elaborar artigos científicos e apresentações cientificas demonstrando seus resultados

\section{Referências}

Alves, J. P., Lins, A. G. T., Barros, T. L. D., \& Farah, B. Q (2017). Comparação entre liberação miofascial e alongamento na flexibilidade em adultos. https://docplayer.com.br/81304347-Comparacao-entre-liberacao-miofascial-e-alongamento-na-flexibilidade-em-adultos.html.

Ajimsha, M. S., Al-Mudahka, N. R., \& Al-Madzhar, J. A. (2015). Effectiveness of myofascial release: systematic review of randomized controlled trials. Journal of bodywork and movement therapies, 19(1), 102-112. https://doi.org/10.1016/j.jbmt.2014.06.001

Braga, D. K. A. P., Marizeiro, D. F., Florêncio, A. C. L., Teles, M. D., Silva, Í. C., Santos-júnior, F. F. U \& Campos, N. G. (2020). Terapia manual no músculo diafragma: efeito na força muscular respiratória e na mobilidade torácica. Manual Therapy, Posturology \& Rehabilitation Journal, 1-5.

Caruso P., Albuquerque A. L. P, Santana P. V., Cardenas, L. Z., Ferreira, J. G., Prina, E. et al. (2015). Diagnostic methods to assess inspiratory and expiratory muscle strength**Study carried out in the Department of Pulmonology, Instituto do Coração - Incor, Heart Institute - University of São Paulo School of Medicine Hospital das Clínicas, São Paulo, Brazil. Jornal Brasileiro de Pneumologia. 41(2), 110-23. https://doi.org/10.1590/S1806-37132015000004474““.

Costa, D., Sampaio, L. M. M., Lorenzzo, V. A. P. D., Jamami, M. \& Damaso, A. R. (2003). Avaliação da força muscular respiratória e amplitudes torácicas e abdominais após a RFR em indivíduos obesos. Revista Latino-Americana de Enfermagem [online]. 2003. 11(2) https://doi.org/10.1590/S01041169200300020000.3

Goetten, D.G. (2018). Efeitos Da Liberação Miofascial: Uma Revisão Da Literatura. 25 f. Monografia (Especialização) - Curso de Fisiologia do Exercicio, Universidade Federal do Paraná, Curitiba, 2018.

Grosu, H. B., Lee, Y. I., Lee, J., Eden, E., Eikermann, M., \& Rose, K. M. (2012). Diaphragm muscle thinning in patients who are mechanically ventilated. Chest, 142(6), 1455-1460. https://doi.org/10.1378/chest.11-1638

Hammer, W.I. (2003). Exame funcional dos tecidos moles e tratamento por métodos manuais: novas perspectivas. 2 ed. Rio de Janeiro: Guanabara Koogan.

Henrique, R. C. ; Pfister, A. P. L. \& Ferreira, R. L. S. (2019). Efeito agudo da liberação miofascial instrumental na modulação autonômica cardíaca em mulheres jovens praticantes de musculação. Conexão Ciência (online), (14),.21-30, 5 abr. Fundacao Educacional de Formiga - FUOM.

Kendall, F. P. ; Mccreary, E. K. \& Provance, P. G. (1995). Músculos: provas e funções com postura e dor. Manole, 453.

McCool, F. D., Benditt, J. O., Conomos, P., Anderson, L., Sherman, C. B., \& Hoppin, F. G., Jr (1997). Variability of diaphragm structure among healthy individuals. American journal of respiratory and critical care medicine, 155(4), 1323-1328. https://doi.org/10.1164/ajrccm.155.4.9105074

Moreno M. A., Catai A. M., Teodori R. M., Borges B. L. A., Cesar M. C. \& Silva E. (2007). Effect of a muscle stretching program using the Global Postural Reeducation method on respiratory muscle strength and thoracoabdominal mobility of sedentary young males. J Bras Pneumol. 33(6):679-686. https://doi.org/10.1590/S1806-37132007000600011.

Moury P. H., Cuisinier A., Durand M., Bosson J. L., Chavanon O., Payen J. F., Jaber S. \& Albaladejo P. (2019). Diaphragm thickening in cardiac surgery: a perioperative prospective ultrasound study. Ann. Intensive Care, 9:50, 1-11. https://doi.org/10.1186/s13613-019-0521-z.

Nair A., Alaparthi, G. K., Krishnan, S., Rai, S., Anand, R., Acharya, V. \& Acharya, P. (2019). Comparison of Diaphragmatic Stretch Technique and Manual Diaphragm Release Technique on Diaphragmatic Excursion in Chronic Obstructive Pulmonary Disease: A Randomized Crossover Trial. Pulmonary medicine, 2019, 6364376. https://doi.org/10.1155/2019/6364376

Novaes, P. A., Sanchez, E. G. M., \& Sanchez, H. M. (2013). Medida das pressões respiratórias máximas em jovens saudáveis antes e após manobra de liberação diafragmática. Movimento \& saúde, Revista Inspirar, jun./jul. (5),1-5. 
Research, Society and Development, v. 10, n. 13, e417101321416, 2021

(CC BY 4.0) | ISSN 2525-3409 | DOI: http://dx.doi.org/10.33448/rsd-v10i13.21416

Pavan, P. G., Pachera, P., Forestiero, A., \& Natali, A. N. (2017). Investigation of interaction phenomena between crural fascia and muscles by using a threedimensional numerical model. Medical \& biological engineering \& computing, 55(9), 1683-1691. https://doi.org/10.1007/s11517-017-1615-0.

Pereira A. S. et al. (2018). Metodologia da pesquisa científica. UFSM.

Rocha, T., Souza, H., Brandão, D. C., Rattes, C., Ribeiro, L., Campos, S. L., Aliverti, A., \& de Andrade, A. D. (2015). The Manual Diaphragm Release Technique improves diaphragmatic mobility, inspiratory capacity and exercise capacity in people with chronic obstructive pulmonary disease: a randomised trial. Journal of physiotherapy, 61(4), 182-189. https://doi.org/10.1016/j.jphys.2015.08.009

Schepens, T., Verbrugghe, W., Dams, K., Corthouts, B., Parizel, P. M., \& Jorens, P. G. (2015). The course of diaphragm atrophy in ventilated patients assessed with ultrasound: a longitudinal cohort study. Critical care (London, England), 19, 422. https://doi.org/10.1186/s13054-015-1141-0

Sutton, B (2016). The Science of Self-Myofascial Release. Retrieved February, (9). 